\title{
An Overview of the NASA Ames Millimeter-Wave Thermal Launch System
}

David D. Murakami, Kevin L. Parkin

NASA Ames Research Center, Moffett Field, CA, 94035

The Millimeter-Wave Thermal Launch System (MTLS) is a beamed-energy propulsion concept being designed at NASA Ames Research Center. This effort is in response to the NASA Office of the Chief Technologist's announcement of the 'Ride the Light' program. Our objective is to produce a design that goes beyond the feasibility analysis level of previous studies and provides a solid foundation for low cost access to space. The MTLS is designed to place a Pegasus XL class payload into Low Earth Orbit (LEO) two times a day. This frequent launch, small payload niche is well suited for the particular advantages and constraints of beamed-energy propulsion, and has the potential to drastically increase access to space by reducing the cost per kilogram of placing payloads into LEO. This paper summarizes the findings of the MTLS study.

\section{Introduction}

The chemical rocket engine is in principle a simple device. It acts by releasing the chemical energy stored in propellants such as hydrogen and oxygen through combustion, then converting that thermal energy into kinetic energy by expansion through a nozzle. As such, it is fundamentally limited by the energy released in combustion reactions and the molecular weight of the products of those reactions. The highest performing conventional propellant combination, liquid oxygen and liquid hydrogen, can produce vacuum specific impulses of around 450 seconds. The design space of current launch vehicles (which tend to be large, multi-stage, and expendable) are defined by these limitations. An entirely new approach may be necessary in order to enable future launch vehicles of radically improved capabilities.

Beamed-energy propulsion (BEP) is an alternative approach that bypasses the energy limitations of chemical propulsion. Instead of relying on a chemical reaction as the energy source, it is supplied externally via a beam of electromagnetic energy produced on the ground. An artist's concept is shown in Figure 1.

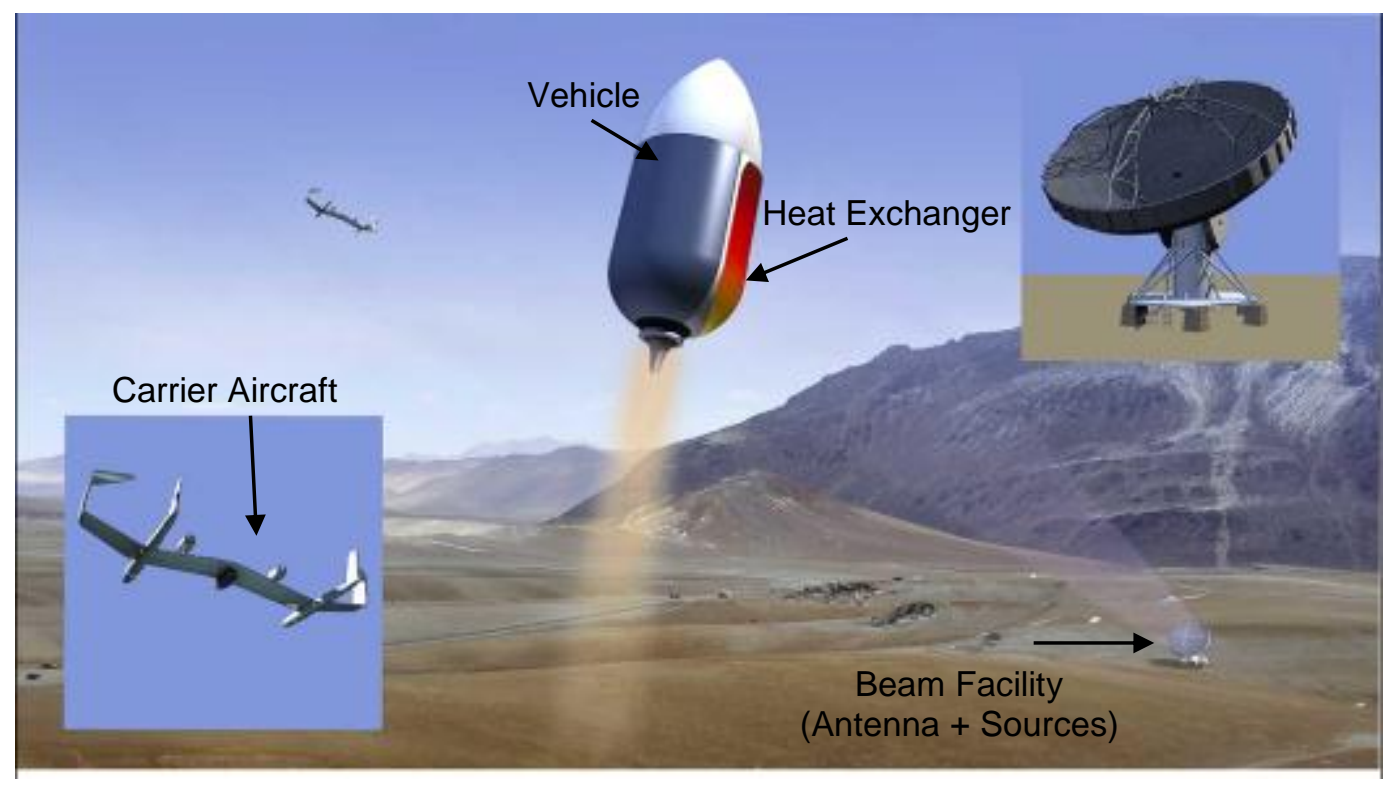

Figure 1: The Beamed-Energy Propulsion Concept

In the concept examined in the MTLS, this energy is absorbed by a heat exchanger which then transfers the energy to the propellant. This decouples the energy source from the working fluid, vastly expanding the design space. For example, a launch vehicle could use only water as propellant, making it very safe and easy to handle. However, the 
most commonly proposed way to take advantage of this decoupling is to use pure hydrogen as the working fluid, which enables specific impulses of around 800 seconds $^{1}$ if the heat exchanger material can operate at $2200 \mathrm{~K}$. With such a significantly increased Isp, it may be possible to build single-stage to orbit vehicles, with enough mass margins left over to permit lower cost fabrication techniques.

The idea of beamed-energy propulsion dates back to 1924 by Tsiolkovsky in his book The Spaceship. Recent investigators include Kare, Myrabo, and Parkin who have studied laser thermal, laser lightcraft, and millimeter-wave thermal concepts. However, its practicality been limited by the difficulty of generating massive amounts of electromagnetic energy and focusing it on a distant target. Concepts using a heat exchanger to absorb electromagnetic energy and transfer it to the working fluid are also constrained by the temperature limits of available materials. The reward for solving these engineering challenges is a propulsion system that circumvents the limitations of conventional chemical rockets - a high Isp ( 800 s), high thrust to weight ratio ( 50$)$ engine becomes possible ${ }^{1}$. Single stage to orbit vehicles, at the far edge of feasibility with conventional propulsion systems, can become practical.

\section{The Millimeter-wave Thermal Launch System}

Advancements in laser and millimeter-wave generation technology over the past several decades and renewed interest in low TRL technology development have resulted in beamed-energy propulsion receiving attention from NASA and DARPA. Recently, two studies investigating various beamed-energy propulsion concepts have been conducted. The Beamed-Energy Propulsion Study ${ }^{2}$ conducted at NASA Glenn considered laser and millimeterwave propulsion for launch vehicles and in-space propulsion, with the objectives of reviewing the state of the art in beamed-energy propulsion, formulating roadmaps for technology development, identifying technology demonstrations to advance the Technology Readiness Level of BEP technology, and assessing concept feasibility. The overall conclusions of this study were that, with long term investment, beamed-energy propulsion for Earth to Orbit applications was feasible and showed distinct advantages over conventional approaches, but very large initial investments in infrastructure were required. The Ride the Light program, run by the NASA Office of the Chief Technologist, has focused on developing power beaming capabilities and has explored other applications, such as inflight recharging of UAVs and ground-to-ground power transmission. The results of this effort are still pending.

In light of this renewed interest, the Millimeter-wave Thermal Launch System (MTLS) was conceived at NASA Ames as a pre-Phase A concept study. The study explored the millimeter-wave thermal propulsion concept in greater depth than previously investigated - investigating details such as site selection and layout, optical train layout, and heat exchanger conceptual design. The MTLS study would thus provide a more solid foundation for bottoms-up feasibility analysis, cost estimation, and identification of technological bottlenecks and trade-offs. Rather than prematurely optimizing for performance, the primary objective of the work was to establish a conservative reference design that could be built with minimal additional technology development and would permit a relatively near-term flyable prototype in the 2020-2030 timeframe.

\section{Reference Mission}

In selecting the reference mission requirements for the study, the traits of beamed-energy propulsion were found to favor frequent small payload launches over infrequent large launches - the MTLS is a bulk cargo or small satellite delivery system, rather than heavy lift or a manned system. This is due primarily to the large capital cost associated with the beam facility, which in this design must generate gigawatts of millimeter-wave energy. This capital cost must be amortized over many flights. A driving cost of the ground facility is beam generation, and the requirements of the beam facility scale roughly linearly with payload mass - a rule of thumb, useful for calibrating intuition, is about $1 \mathrm{MW}$ of beam energy per kilogram of payload ${ }^{3}$. Gyrotrons, the current state of the art in millimeter-wave sources, can produce 1-2 megawatts of power each, and can be obtained for approximately $\$ 2$ per watt. The other driving cost is the transmission system; the aperture required to focus a millimeter-wave beam onto a launch-vehicle sized target from hundreds of kilometers away is quite large, requiring a dish the size of the largest radio telescopes built ( 100 meter diameter). These factors favor a small launch vehicle with a short powered ascent phase in order to reduce start-up costs. A Pegasus XL class payload was judged to be large enough to maintain applicability to a broad range of missions, but small enough to result in manageable requirements on the beam facility.

The flight segment of the MTLS is developed around an air-launched, single stage to orbit concept of operations. The launch vehicle is lifted to its launch altitude by a carrier aircraft, while it is tracked by the beam facility. At 
launch, the vehicle is dropped from the carrier aircraft and power transmission from the beam facility to heat the launch vehicle heat exchanger begins. The vehicle simultaneously begins to pump propellant through the heat exchanger and begins to produce thrust. The powered ascent lasts only a few minutes and covers a few hundred kilometers of downrange distance. The downrange distance constraint is set by the feasible size of aperture at the beam facility - this size limit was set by comparison to existing large radio telescopes. After beam cut-off, the vehicle coasts to apogee, at which point the payload and vehicle separate. The payload contains a kick motor to circularize its own orbit at apogee, while the expendable launch vehicle then continues to a re-entry. Figure 2 depicts a preliminary trajectory for the powered portion of the flight. At apogee, a small kick motor is then used to circularize the orbit. The trajectories were analyzed with the POST II targeting and optimization software. An elevation angle constraint is intended to prevent excessive atmospheric absorption of the beam, as well as for antenna related mechanical reasons. The air launch, apart from its operational advantages of enabling any launch azimuth around the fixed ground facility, also helps to meet this elevation angle constraint.

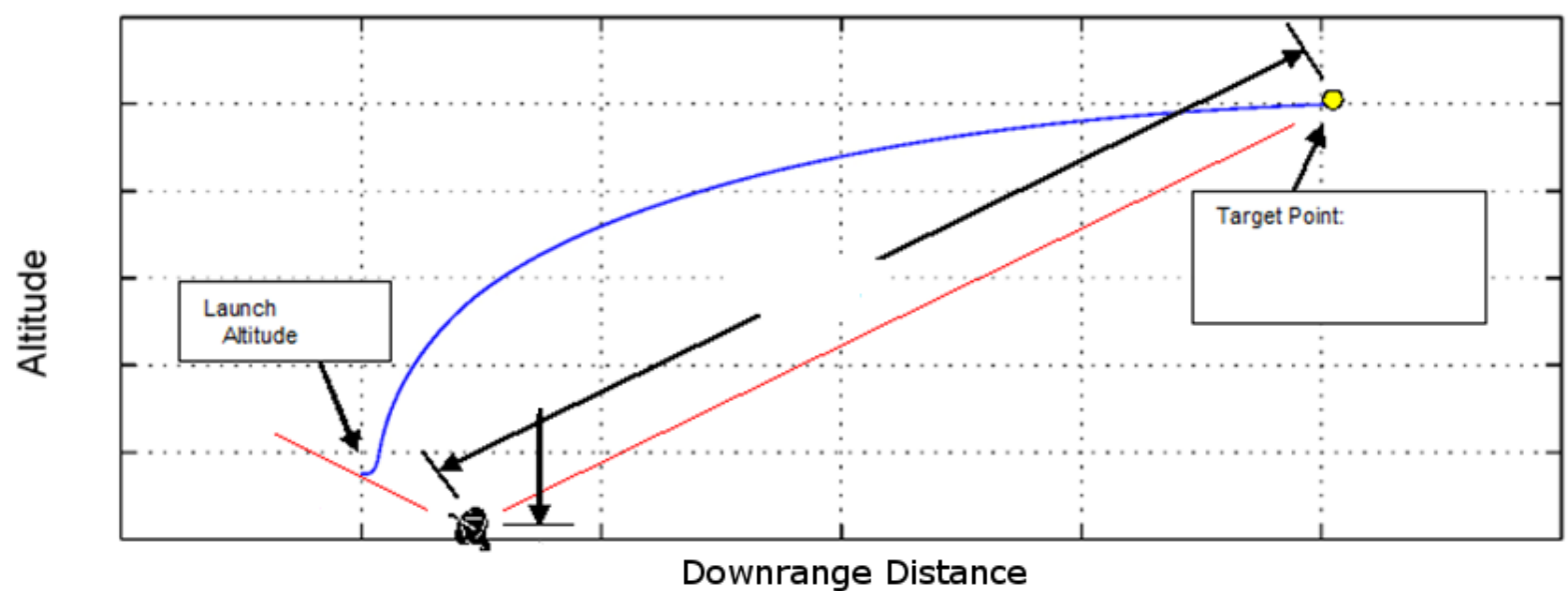

Figure 2: Preliminary Trajectory

This preliminary trajectory was used primarily for its simplicity and ease of analysis with the tools and expertise at our disposal. More complex trajectories can be considered that optimize different system parameters. For example, Figure 3 shows a recurve trajectory that lengthens the acceleration distance of the launch vehicle and keeps it closer to the beam facility during the powered phase. This results in more propellant consumption than the baseline trajectory, but reduces peak accelerations to almost half the original value, reduces propellant mass flow rates by a quarter, and reduces the required power output of the ground station by $9 \%$. 


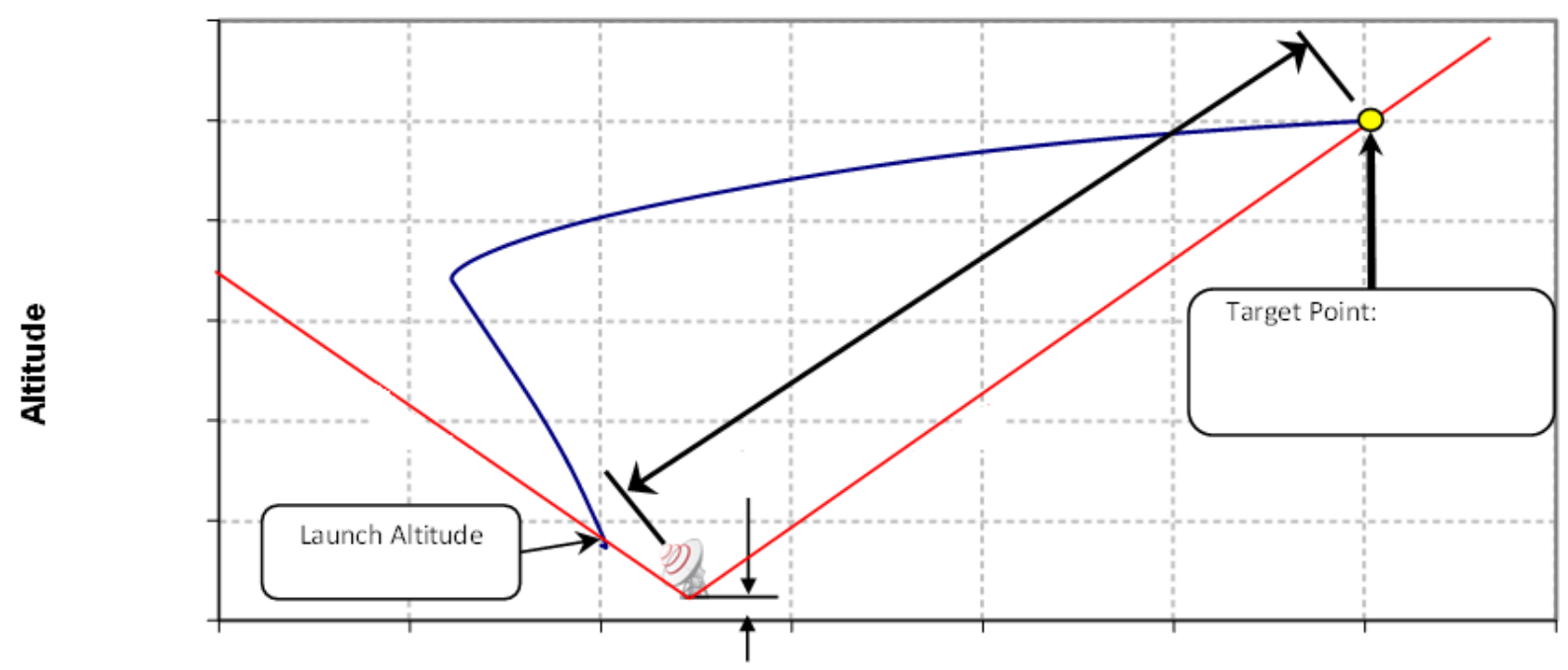

Downrange Distance

Figure 3: Reduced G-Load Trajectory

\section{Baseline Vehicle}

The simplest and most conservative approach to designing the launch vehicle for the MTLS is to take our intuition of what a launch vehicle should be (i.e. a flying propellant tank) and replace the engine with a beamed-energy propulsion system. This is somewhat equivalent to replacing the combustion chamber of a chemical engine with a heat exchanger. This produces a configuration with the least amount of technical uncertainty, since it shares the most in common with well-established launch vehicle designs and can be analyzed in a similar fashion. A figure depicting the launch vehicle is provided in Figure 4. 


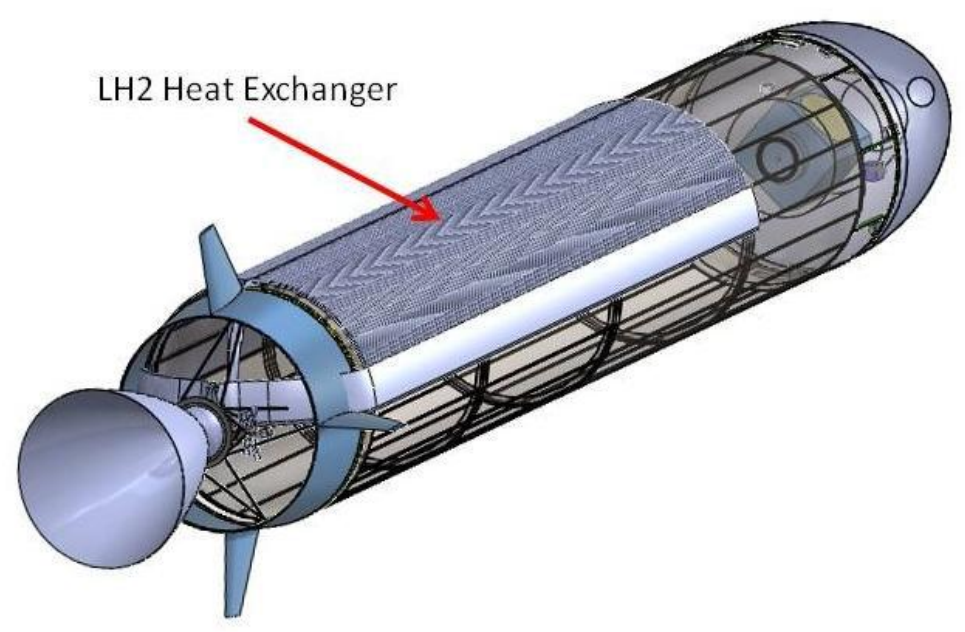

Figure 4: Launch Vehicle Overview

Because of the low density of liquid hydrogen (only about $70 \mathrm{~kg} / \mathrm{m}^{3}$ ), tank mass is the main factor in the launch vehicle mass budget. Drawing inspiration from the Atlas and Centaur vehicles, the MTLS vehicle utilizes a titanium pressure-stabilized tank, incorporating additional structural stiffening elements, maintained slightly above atmospheric pressure. The wall thickness is thinner than that of the Atlas or Centaur tanks, and comparable to advanced composite overwrapped pressure vessels. A fin assembly is included at the rear for aerodynamic stability during the air-launch procedure. An expander cycle turbopump system is used, powered by hot propellant bleed from the heat exchanger exit.

This expendable vehicle is intended to be air-launched from an unmanned carrier UAV. Aside from the air launch and a small payload apogee circularization motor, which are necessary due to beam facility constraints, this is a single stage to orbit vehicle. Some of the technical parameters of the baseline vehicle are listed in Table 1below. A standard mass margin is included in the dry mass.

\section{Table 1: Key Technical Parameters}

\begin{tabular}{|l|r|}
\hline Propellant & Hydrogen \\
\hline Heat Exchanger Exit Temperature (K) & $1800-2200$ \\
\hline Vacuum Specific Impulse (s) & 700 \\
\hline Vacuum Thrust (kN) & $200-300$ \\
\hline Nozzle Expansion Ratio & 30 \\
\hline Dry Mass (kg) & 2000 \\
\hline Propellant Mass (kg) & 3000 \\
\hline
\end{tabular}

The relatively low vacuum Isp is caused by the low expansion ratio nozzle - this in turn was set by the requirement for air-launch operation with low plenum pressure. Large expansion ratios at low altitudes lead to flow separation in a bell nozzle (usually at $25-40 \%$ of ambient pressure) and potentially dangerous unsteady forces that could destroy the vehicle. Very high chamber pressures or novel nozzle designs (such as a plug nozzle) are required to allow vacuum-optimized nozzles to run at low altitudes. Feasibility and clarity of analysis, rather than performance, were the main objectives of the MTLS study, so the performance penalty of using a simple bell nozzle was accepted.

The design process resulted in a $\sim 5$ metric ton wet mass vehicle that could deliver a small payload with a single stage. This can be compared to the Pegasus XL, a small, air launched, current vehicle that can deliver 443 kilograms to orbit with a 23 metric ton wet mass using three solid stages. Due to the MTLS vehicle's significant wet mass savings, it was noted that the MTLS vehicle could also perform well as an upper stage to a combined 
chemical and beamed energy launch vehicle - however, this application is outside the scope of the study.

\section{Heat Exchanger}

On the vehicle, the major technological challenge is the heat exchanger. The nearest analogies for the requirements of the MTLS come from air-breathing hypersonic vehicles, nuclear thermal rockets, and regeneratively cooled rocket engines. The key metrics for heat exchanger materials are the strength to density ratio, thermal conductivity, and melting point. Refractory metals such as tungsten have been used in similar applications, but the development of woven Carbon/Carbon and Carbon/Silicon Carbide heat exchangers may enable high thrust to weight beamedenergy propulsion systems.

In wireless energy transmission, where beam divergence due to diffraction must be minimized to keep receiving and transmitting aperture sizes reasonably small, Gaussian beam profiles are commonly used. A top-hat profile (constant intensity over the entire receiver) would be preferable in minimizing the area and mass of the heat exchanger, but such a profile would require an even larger and more complex ground station. Gaussian profiles require a large difference between peak and minimum intensity if a reasonable amount of the total energy in the beam is to be captured. In the heat exchanger analysis, the beam width (defined as where intensity drops to $1 / \mathrm{e}^{2}$ of the value at the peak) was assumed to match the heat exchanger dimensions. This is depicted in Figure 5. This was taken as the baseline case for the preliminary design.

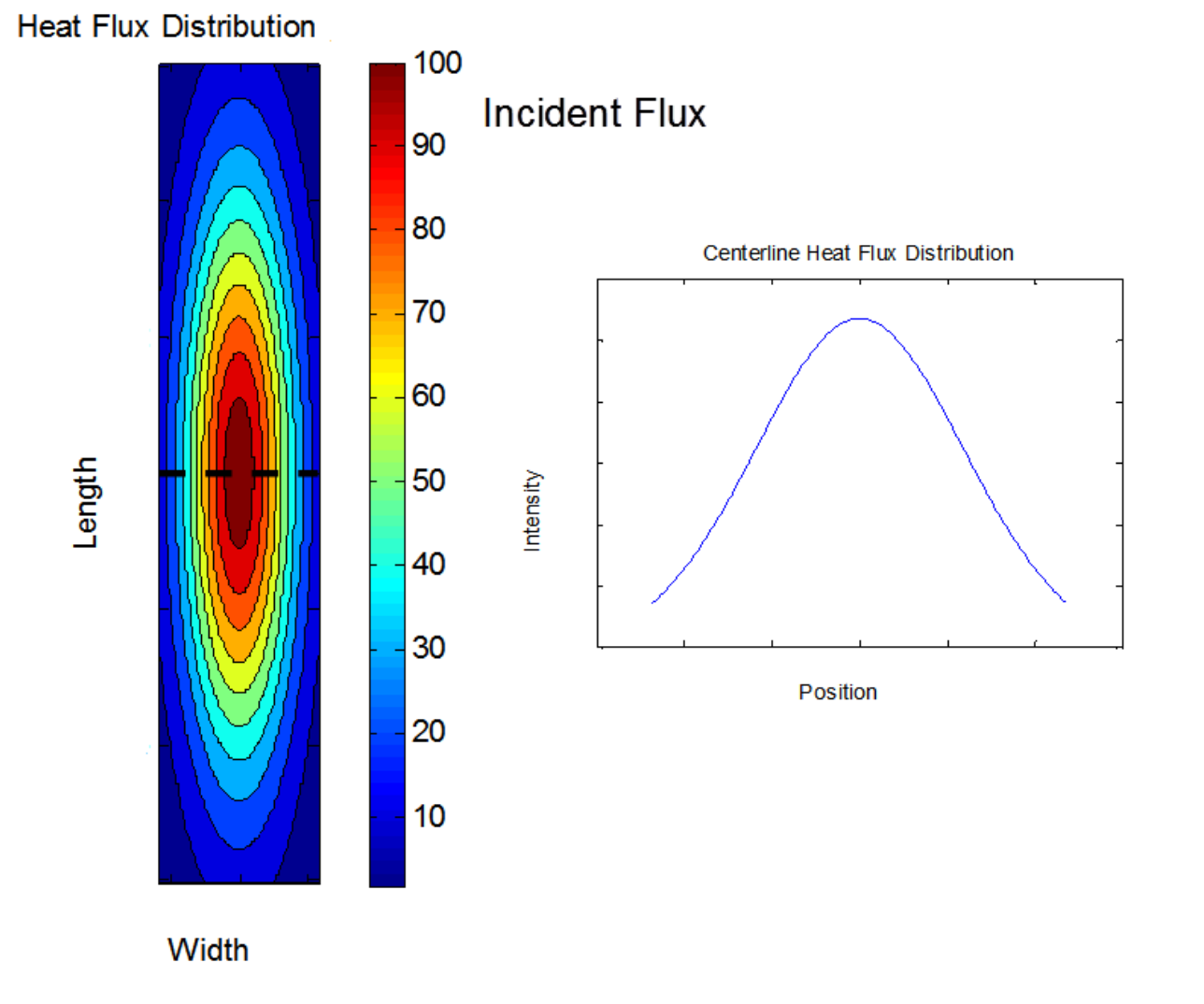

Figure 5: Heat Exchanger Heat Flux Distribution

In the MTLS launch vehicle the heat exchanger is conformal to the tank, as opposed to an entirely separate structure. For comparison, a vehicle concept with an entirely separate structure is depicted in Figure 6. 


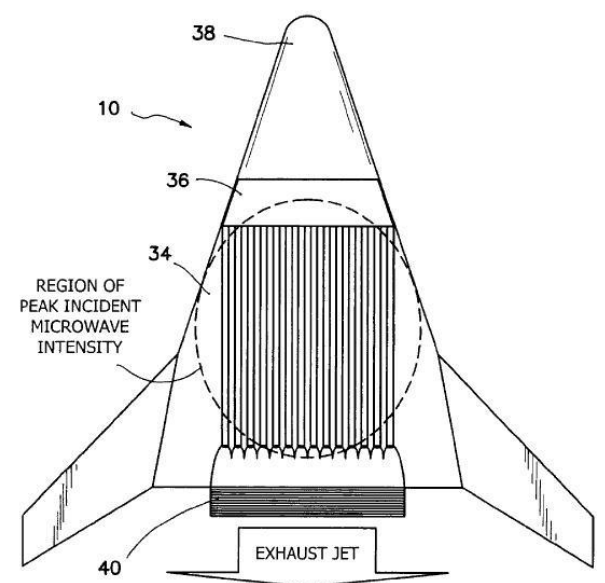

\section{Figure 6: Vehicle Based on X-33 Aeroshell}

The tank conformal configuration is simple and easy to analyze, but the maximum heat exchanger size is limited to half of the tank area. This drives a coupling between payload mass (which drives the dimensions of the tank) and beam intensity at the target (which drives antenna dimensions on the ground). The end result is higher required heat fluxes for the conformal case than the separate case, but the additional complexity and uncertainty associated with separate heat exchanger structure were deemed undesirable for the current study.

The layout of the heat exchanger channels was also an interesting problem. Three layout concepts are shown in Figure7. The simplest approach ("straight through") would place all the channels running down the length of the vehicle, with the cold inlet at the front and the hot outlet at the back (nearest the nozzle). The "Fishbone" configuration with the inlet down the centerline and outlet at the sides has the flow travel circumferentially rather than axially. This delivers the coolest propellant at the highest heat flux region and also reduces pressure losses. The "Curved Fishbone" can reduce peak wall temperatures by introducing additional curvature near the inlet. Peak wall temperatures tend to occur near the mid-channel region, where heat flux is still high (which dictates the temperature difference between inner and outer wall) but the propellant has warmed significantly (raising the temperature of the inner wall). Near the center, the heat flux is high but offset by the low fluid temperatures, while near the sides the heat flux has decayed to an almost insignificant value and the wall temperature is essentially the same as the fluid temperature. The curvature in the "Curved Fishbone" acts to alter the angle of incidence of the beam (and thus its intensity due to the cosine law) at this critical mid-channel region. For the particular parameters used in the MTLS point design, the "Fishbone" was found to have acceptable performance and was selected.

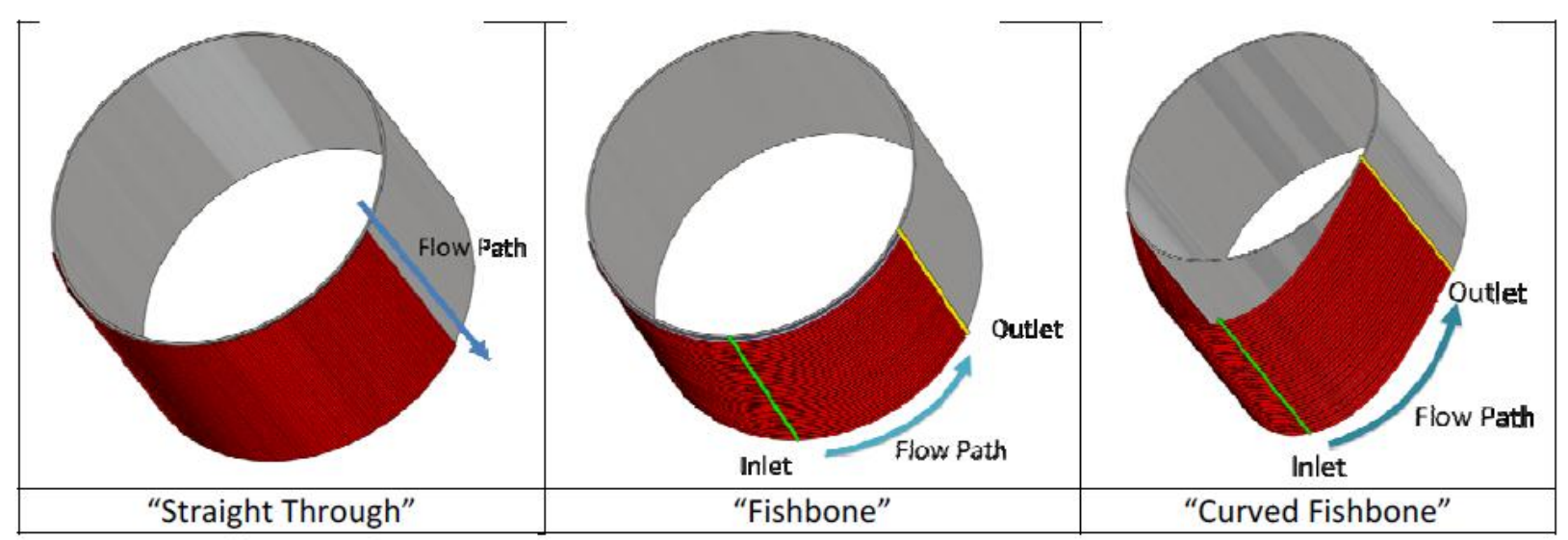

Figure 7: Heat Exchanger Flow Layouts 
The results of a quasi 1D heat transfer analysis lead to dimensions that are similar those of heat exchangers developed for air-breathing hypersonic vehicles and regeneratively cooled engines. The peak wall temperatures are more typical of radiatively cooled engines.

\section{Beam Facility}

The major distinction between beamed-energy launch concepts and conventional launch vehicles is the significant power beaming infrastructure on the ground. Although extensive, the beam facility has no limitations on mass, is not affected by launch vehicle failures, and is completely reusable. The beam facility is a capital cost that is amortized over the many launches of the MTLS.

Beamed energy propulsion concepts generally operate in either laser or millimeter-wave frequencies, due to the absorption characteristics of the atmosphere. Within the millimeter-wave region, certain frequencies are unsuitable for wireless energy transmission due to absorption by water vapor. Some typical data on the transmission properties of millimeter waves is provided in Figure 88. The MTLS beam facility is designed to operate in the $110 \mathrm{GHz}$ to 170 $\mathrm{GHz}$ window.

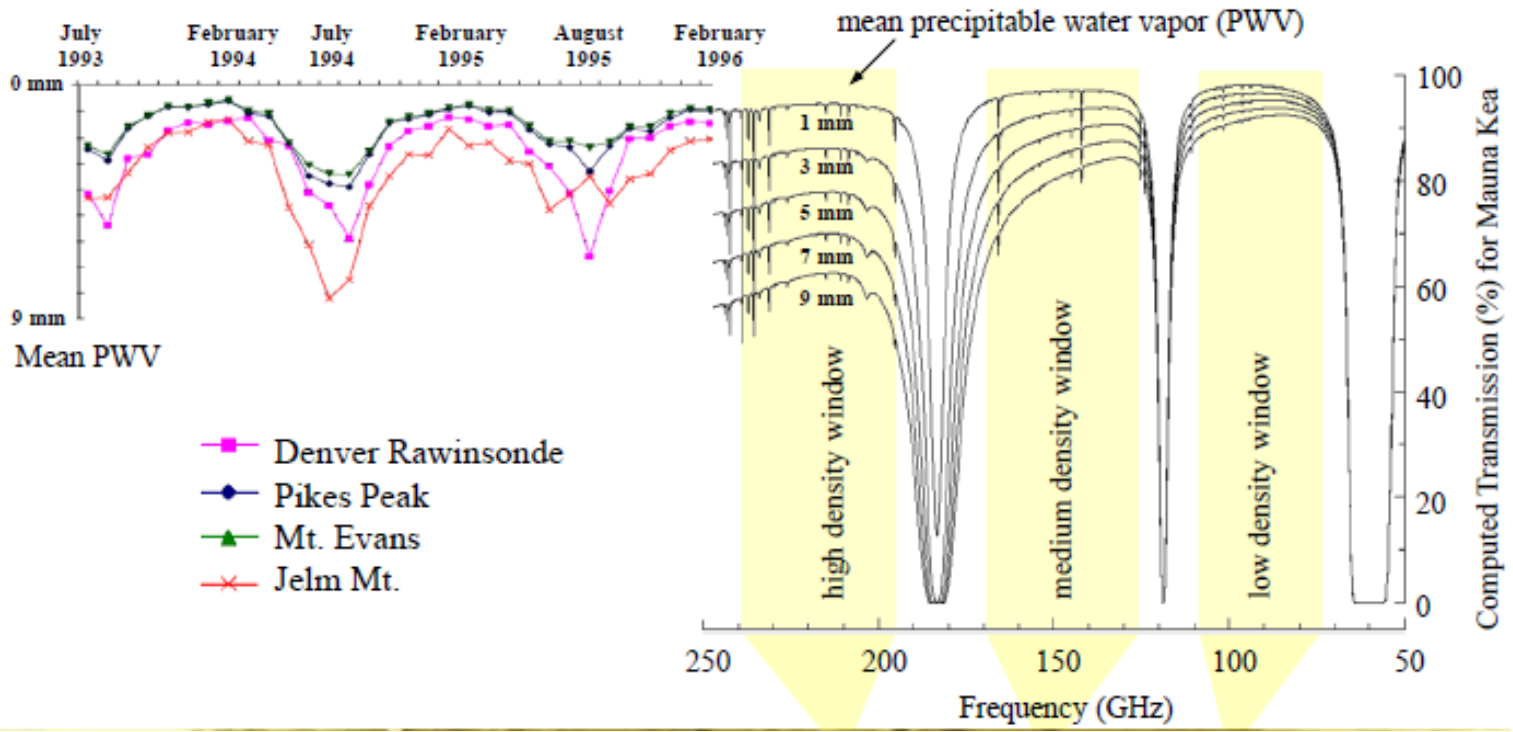

Figure 8: Atmospheric Transmission Window

The beam generation technology chosen for the MTLS baseline is the gyrotron, which is a millimeter-wave source developed primarily for electron cyclotron resonance heating in nuclear fusion research. Units at the megawatt power level are commercially available, and have been used in previous beamed-energy propulsion tests ${ }^{5}$. A 2.2 MW, $170 \mathrm{GHz}$ system is under development for ITER ${ }^{7}$, the world's largest experimental tokamak fusion reactor. The millimeter wave sources baselined for this study were based on the requirements for the ITER system.

The beam facility consists of power storage, gyrotron-based millimeter-wave beam production, beam combination, and transmission systems. The electrical power used during powered flight is several gigawatts produced over a few minutes, a value far too large to be supplied directly by a utility. From an analysis of various energy storage concepts, banks of flywheels were selected. These charge over several hours in between launches, and rapidly discharge during flight. The discharged power is consumed by a network of gyrotrons mounted in trailers, which produce thousands of individual beams at slightly differing frequencies that are combined using a diffraction grating in a spectral beam combining setup. This combined beam is sent through a series of mirrors to an offset parabola primary antenna, where it is then directed towards the vehicle. For the MTLS point design, the beam facility is split into two mirrored facilities due to power handling limitations on the components of the combined beam. 


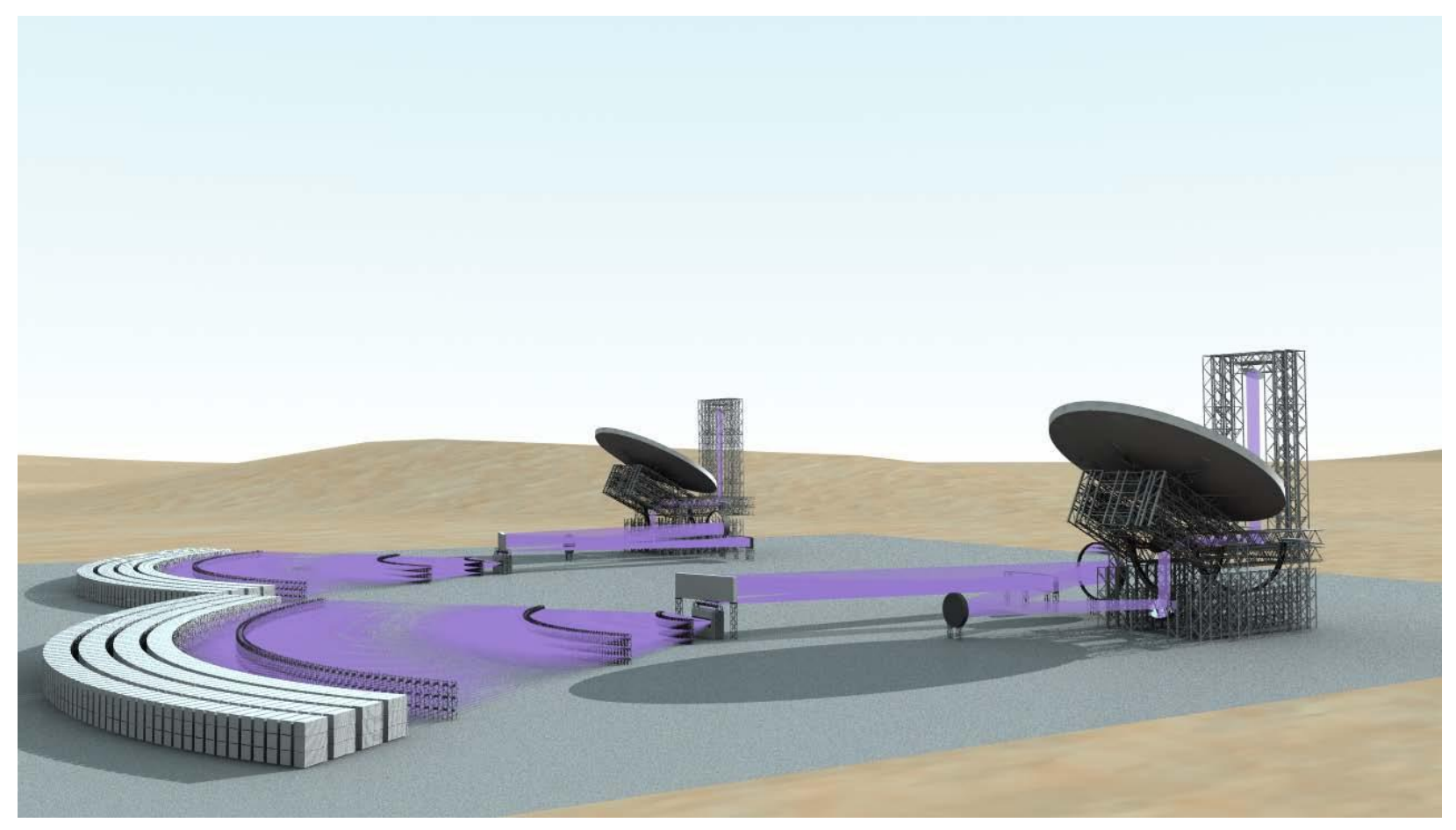

Figure 8: Beam Facility Artist's Concept (Credit: NASA Ames and Lockheed Martin)

In finding suitable locations for the beam facility, atmospheric attenuation of the beam is a major consideration. Dry, high altitude locations are preferred. Within the continental United States, the eastern Sierra Mountains and sections of the southwest have favorable propagation characteristics. Hawaii is also a favorable site due its latitude and remoteness.

Spectrum management is also a significant consideration. Although the 110 to $170 \mathrm{GHz}$ range is not heavily used, sections have been allocated to radio astronomy, fixed, mobile, and satellite communications applications. The current regulatory structure is not currently well adapted to deal with beamed-energy propulsion, and negotiation with regulatory bodies such as the International Telecommunication Union, Federal Communications Commission, and National Telecommunications and Information Administration will be required. The beam facility itself is considered unlikely to be a major source of interference due to its remote location and extremely highly directional beam, but reflections from the launch vehicle may be problematic. The short time span of the event should also be taken into consideration. The Joint Space Operations Center and Laser Clearinghouse are both Department of Defense run organizations to prevent ground based hazards such as lasers from interfering with satellites, and close cooperation with these groups will be required.

\section{Summary}

With the current means at our disposal, achieving orbit is an exceptionally complex, difficult, and expensive task. There are certainly many economic, historical, and political factors that go in to this; however, from the engineering perspective, a chief technical reason is that chemical rockets are a mature technology - well studied, well understood, and optimized to the point of being near-ideal thermodynamic devices. No revolutionary improvements in basic performance are possible, as they are fundamentally limited in specific impulse, a key performance parameter, by the thermochemical properties of the propellants used. To radically improve access to space, a radically different basic approach may be required. The MTLS study has examined the millimeter-wave thermal propulsion concept and produced a design for a near-term, small payload launch system. This design provides detail useful for feasibility analysis and future optimization and refinement work. The ground infrastructure required is extensive, but even this study stressing simplicity and ease of analysis results in a simpler and higher performing launch vehicle than is currently possible. 


\section{Acknowledgments}

The authors would like to thank foremost the Lockheed Martin and NASA Ames personnel of the MTLS study team for their hard work and dedication. This work was supported under contract NAS2-02090 with Lockheed Martin and NASA Grant and Cooperative Agreement NNX09AF52A with Carnegie Mellon University. The support of the NASA Ames Graduate Co-op Research Program is gratefully acknowledged.

\section{References}

[1] Parkin, K., "The Microwave Thermal Thruster and its Application to the Launch Problem," Ph.D. Dissertation, Aeronautics and Astronautics Dept., California Institute of Technology, Pasadena, CA, 2006

[2] George, P., and Beach, R., "Beamed-Energy Propulsion (BEP) Study,” NASA/TM-2012-217014

[3] Kare, J. T., "Laser-Powered Heat Exchanger Rocket for Ground-to-Orbit Launch," Journal of Propulsion and Power, Vol. 11, No. 3, 1995, pp. 535-543

[4] Parkin, K., "Microwave heat-exchange thruster and method of operating the same." U.S. Patent 6,993,898, issued Feb 7, 2006

[5] Oda, Y., Nakagawa, T., "An observation of plasma inside of microwave boosted thruster." Second International Symposium on Beamed Energy Propulsion, Sendai, Japan. Conference Proceedings of the American Institute of Physics. 